\title{
Study on Advanced Frequency Estimation Technique using Gain Compensation
}

\author{
Chul-Won Park ${ }^{\dagger}$, Dong-Kwang Shin*, Chul-Hwan Kim**, \\ Hak-Man Kim*** and Yoon Sang Kim****
}

\begin{abstract}
Frequency is an important operating parameter for the protection, control, and stability of a power system. Thus, it must be maintained very close to its nominal frequency. Due to the sudden change in generation and loads or faults in a power system, however, frequency deviates from its nominal value. An accurate monitoring of the power frequency is essential for optimum operation and prevention of wide area blackout. Most conventional frequency estimation schemes are based on the DFT filter. In these schemes, the gain error could cause defects when the frequency deviates from the nominal value. We present an advanced frequency estimation technique using gain compensation to enhance the DFT filter-based technique. The proposed technique can reduce the gain error caused when the frequency deviates from the nominal value. Simulation studies are performed using both the data from EMTP-RV software and the user-defined arbitrary signals to demonstrate the effectiveness of the proposed algorithm. Results show that the proposed algorithm achieves good performance under both steady state tests and dynamic conditions.
\end{abstract}

Keywords: DFT filter, EMTP-RV, Gain compensation, Frequency, Frequency estimation, Nominal frequency, Wide area blackout

\section{Introduction}

Frequency is an important operating parameter for the protection, control, and stability of a power system [1]. As a key index of power quality, frequency can be indicative of system abnormal conditions and disturbances [2]. Due to the sudden change in generation and loads or faults in power system, frequency is expected to deviate from its nominal value. Thus, the power frequency must be maintained very close to its nominal frequency. In addition, frequency measure devices and frequency tracking techniques should be fast and accurate in determining the frequency [3].

Recently, time-synchronized phasor and frequency measurement methods for fault disturbance recorders, phasor measurement units, and intelligent power system information units have attracted attention. In the United States, a frequency monitoring network by capable of careful monitoring of frequency and frequency deviation

\footnotetext{
Corresponding Author: Department of Electrical Engineering, Gangneung-Wonju National University, Korea. (cwpark1@gwnu.ac.kr)

* Department of Information and Communication Engineering, Sungkyunkwan University, Korea. (shin8165@naver.com)

** Department of Information and Communication Engineering, Sungkyunkwan University, Korea. (hmwkim@hanmail.net)

*** Department of Electrical Engineering, University of Incheon, Korea. (hmkim@incheon.ac.kr)

**** School of Computer and Science Engineering, Korea University of Technology and Education, Korea. (yoonsang@kut.ac.kr)

Received: October 12, 2010; Accepted: February 15, 2011
}

with high precision to a common reference of the GPS has become an important component of wide area measurements in a power system [4-7]. In Korea, the wide area measurement system has been developed last year [8-11].

After the microprocessor was produced, numerous measurement and tracking techniques for frequency and frequency deviation were reported during the past three decades. Most of these techniques process the sampled and digitized values of the system voltage to frequency and frequency deviation measurement [12-13]. However, because most conventional frequency estimation techniques are based on the DFT filter, the gain error for magnitude changes could cause defects when the power system frequency deviates from the nominal value [1417].

To improve the performance of DFT filter-based techniques, we presents an advanced frequency estimation technique using gain compensation. To demonstrate the performances of the proposed algorithm, we use EMTP-RV simulation data and user-defined arbitrary signals, sampled with $720 \mathrm{~Hz}$ per cycle. The proposed technique can reduce the gain error caused by the deviation of the power system frequency from the nominal value. This paper is organized as follows: In Section 2, advanced frequency estimation algorithm with gain compensation is reviewed. Simulation studies are introduced and discussed in Section 3. In Section 4, results from the proposed algorithm are given. 


\section{Advanced Frequency Estimation Algorithm}

\subsection{Frequency Estimation Technique using Phase Angle Difference of Two Phasors}

Correlating one cycle of reference fundamental frequency cosine and sine waveforms with the voltage signal, the fundamental frequency real and imaginary components $V_{r 1}^{12}(k)$ and $V_{i 1}^{12}(k)$, present in a voltage signal at any sampling instant for $\mathrm{N}=12$ are given by

$$
\begin{aligned}
V_{r 1}^{12}(k)= & \frac{2}{12}\left[V_{k}-V_{k-6}+0.5\left(V_{k-10}-V_{k-8}-V_{k-4}+V_{k-2}\right)\right. \\
& \left.+0.866025404\left(V_{k-11}-V_{k-7}-V_{k-5}+V_{k-1}\right)\right] \\
V_{i 1}^{12}(k)= & \frac{2}{12}\left[V_{k-9}-V_{k-3}+0.5\left(V_{k-11}+V_{k-7}-V_{k-5}-V_{k-1}\right)\right. \\
& \left.+0.866025404\left(V_{k-10}+V_{k-8}-V_{k-4}-V_{k-2}\right)\right]
\end{aligned}
$$

where $V_{k-n}$ is the sample at $(k-n+N)$ th sampling instant.

To extract the fundamental frequency component using DFT filter, the real and imaginary parts computed using samples corresponding to the $n$th data window can be used to represent the signal in phasor form by the following Eq. (3):

$$
\overline{V_{n}}=V_{r n}+j V_{i n}
$$

where $V_{r n}$ and $V_{i n}$ are the real and imaginary parts computed using samples from the $n$th data window, respectively. Similarly, $V_{r n+1}$ and $V_{i n+1}$ are the real and imaginary parts computed using samples from the $(n+1)$ th data window, respectively.

The phase angle difference, $\left(\theta_{n+1}-\theta_{n}\right)$, represents the rotation of the phasors as the data window is advanced by one sample. Finally, the frequency estimation $\widehat{f}$ can be obtained by the following Eq. (4):

$$
\widehat{f}=\frac{\theta_{n+1}-\theta_{n}}{\frac{2 \pi}{F_{s}}}
$$

where $F_{s}$ and $\hat{f}$ are the sampling frequency and the frequency estimation, respectively.

\subsection{Advanced Frequency Estimation Algorithm with Gain Compensation}

Consider that sinusoidal voltage signal can be expressed by Eq. (5):

$$
v(n)=A \cos \left(2 \pi n \frac{f}{f_{s}}+\theta\right)
$$

where $A$ and $\theta$ are the magnitude and the phase angle, respectively.

By applying the frequency response of cosine filter to $v(n)$, Eq. (5) can be into Eq. (6). Similarly, frequency response of sine filter can be represented in Eq. (7):

$$
\begin{aligned}
& v_{c}(n)=A_{c} \cos \left(2 \pi n \frac{f}{f_{s}}+\theta\right) \\
& v_{s}(n)=A_{s} \sin \left(2 \pi n \frac{f}{f_{s}}+\theta\right)
\end{aligned}
$$

where, $A_{c}=A\left|H_{c}(f)\right|, A_{s}=A\left|H_{s}(f)\right|, \hat{\theta}=\theta-\pi \frac{f(N-1)}{f_{s}^{2}}$.

Subsequently, the ratio of magnitude of $v(n)$ can be expressed mathematically as

$$
\frac{A_{c}}{A_{s}}=\frac{\left|H_{c}(f)\right|}{\left|H_{s}(f)\right|}=\frac{\tan \left(\frac{\pi f}{f_{s}}\right)}{\tan \left(\frac{\pi f_{0}}{f_{s}}\right)}
$$

From Eq. (8), the frequency $f$ can be expressed as

$$
f=\frac{f_{s}}{\pi} \tan ^{-1}\left(\tan \left(\frac{\pi f_{0}}{f_{s}}\right) \frac{A_{c}}{A_{s}}\right)
$$

where $f_{0}$ is the fundamental frequency component.

From the combination of Eq. (6) and Eq. (7), an elliptic equation is expressed as

$$
\left(\frac{v_{c}(n)}{A_{c}}\right)^{2}+\left(\frac{v_{s}(n)}{A_{s}}\right)^{2}=1
$$

Using the output of cosine and sine filter for $v(n)$ and $v(n-1)$, Eq. (10) can be expressed as

$$
\left[\begin{array}{cc}
v_{c}{ }^{2}(n) & v_{c}{ }^{2}(n) \\
v_{s}{ }^{2}(n-1) & v_{s}{ }^{2}(n-1)
\end{array}\right]\left[\begin{array}{c}
1 / A_{c}{ }^{2} \\
1 / A_{s}{ }^{2}
\end{array}\right]=\left[\begin{array}{l}
1 \\
1
\end{array}\right]
$$

Based on the arrangement of Eq. (11), the ratio of $A_{c}$ to $A_{s}$ can be expressed as

$$
\frac{A_{c}}{A_{s}}=\sqrt{\frac{v_{c}^{2}(n)-v_{c}^{2}(n-1)}{-v_{s}^{2}(n)+v_{s}^{2}(n-1)}}
$$

Finally, substituting Eq. (12) to Eq. (9), we obtain an 
equation for frequency estimation with gain compensation

$$
f=\frac{f_{s}}{\pi} \tan ^{-1}\left(\tan \left(\frac{\pi f_{0}}{f_{s}}\right) \sqrt{\frac{v_{c}^{2}(n)-v_{c}^{2}(n-1)}{-v_{s}^{2}(n)+v_{s}^{2}(n-1)}}\right.
$$

Fig. 1 shows the flowchart of the advanced frequency estimation algorithm.

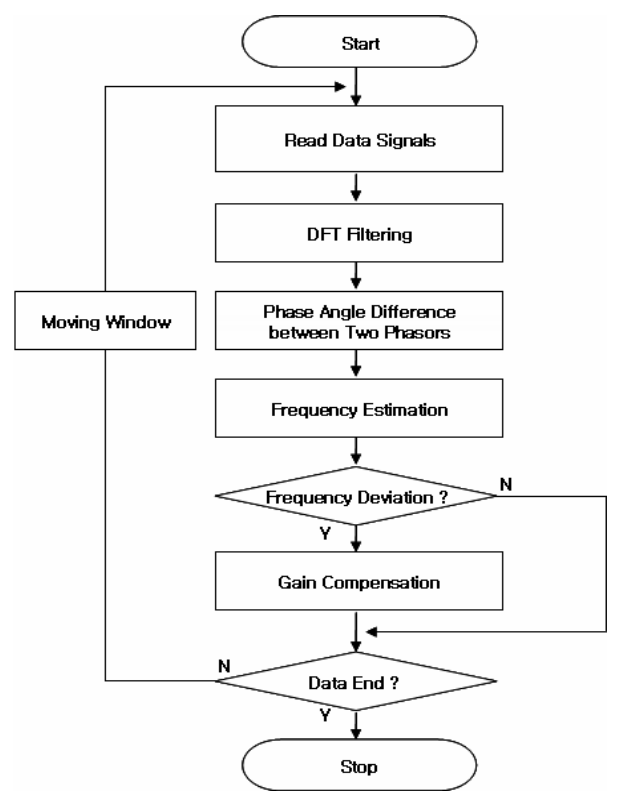

Fig. 1. Flowchart of the advanced frequency estimation algorithm

\section{Simulation Studies}

Comprehensive evaluation of the proposed frequency estimation algorithm was carried out by conducting several test cases using EMTP-RV modeling data and user-defined arbitrary signals.

\subsection{Evaluation using EMTP-RV modeling data}

The test voltage data were obtained from an EMTP-RV simulation sampled with $12 \mathrm{~S} / \mathrm{C}$. The $765 \mathrm{kV}$ T/L system in Korea is simulated by the EMTP-RV software. Fig. 2 shows the power system model used for the simulation.

The modeling for the governor and exciter of Uljin N/P and Dangjin T/P was obtained based on real data, and T/L between Shin-gapyung and Shin-ansung was simulated based on the places where the construction will be conducted. From the $765 \mathrm{kV}$ T/L system shown in Fig. 2, the voltages of six regions, namely, Dangjin, Shin-seosan, Shin-ansung, Shin-gapyung, Shin-taebaek, and Uljin, were measured. Measurements were performed under the parameters of $100 \mathrm{MW}$ load shedding and $400 \mathrm{MW}$ load shedding at Dangjin T/P.

Fig. 3 shows the three phase voltage signals on $100 \mathrm{MW}$

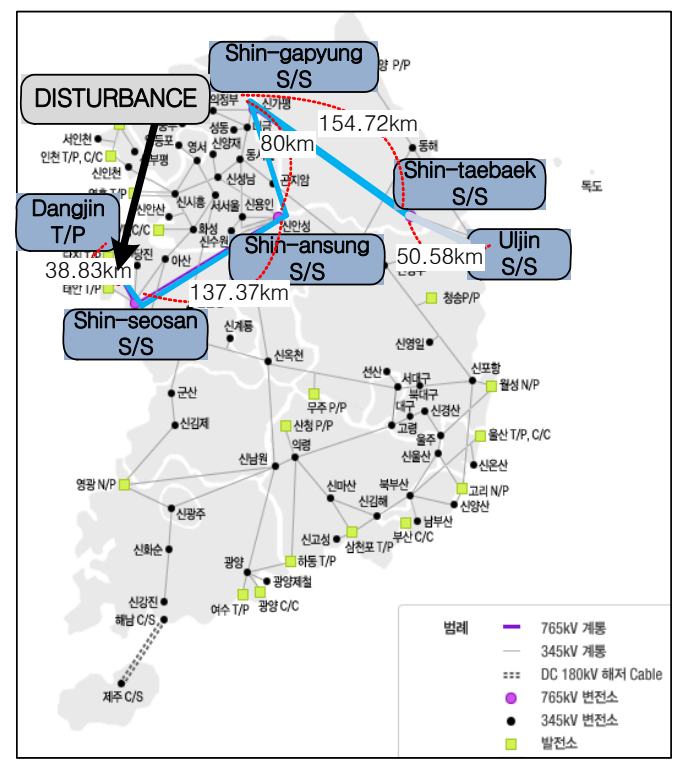

Fig. 2. Power system model of EMTP-RV

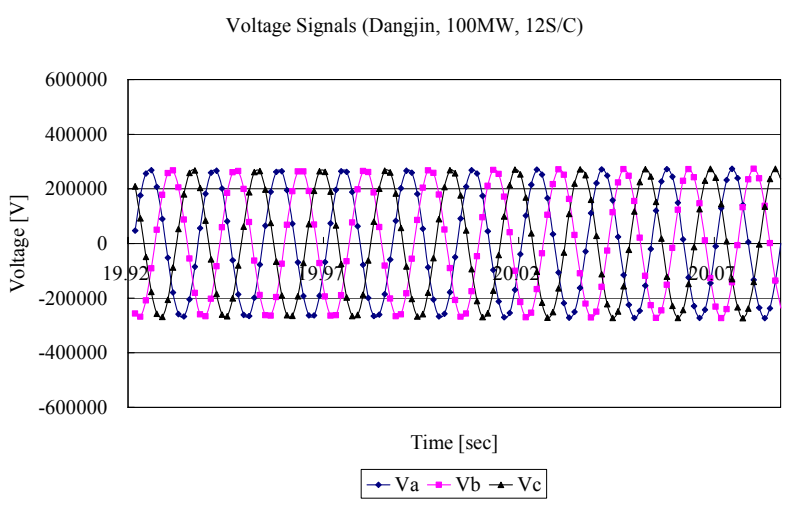

(a)

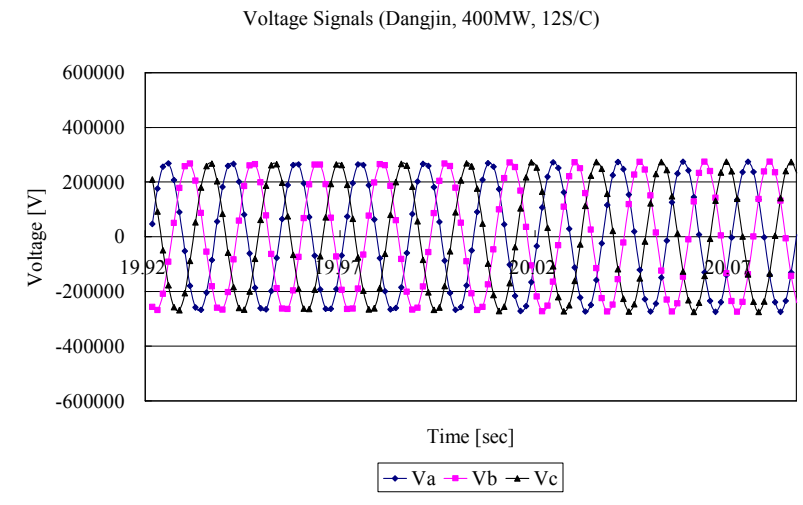

(b)

Fig. 3. Three phase voltage signals of Dangjin T/P

load shedding and $200 \mathrm{MW}$ load shedding at Dangjin T/P in approximately $20 \mathrm{sec}$.

Fig. 4 shows the computed frequency by EMTP-RV and the frequency of each local area estimated during $100 \mathrm{MW}$ load shedding at Dangjin. Fig. 5 shows the computed 


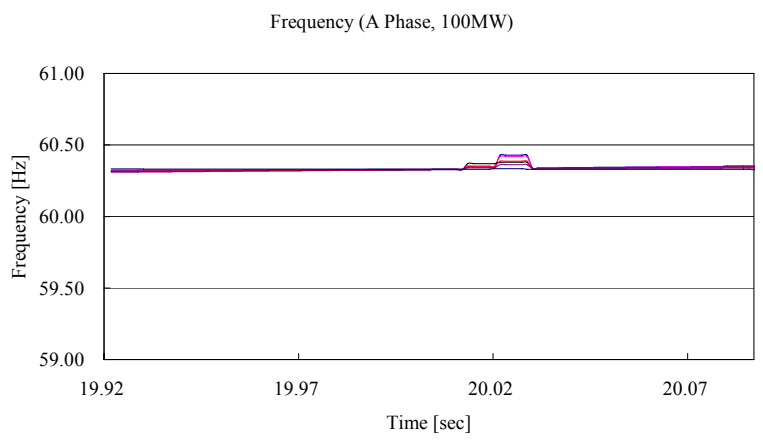

— Dangjin — Shin-seosan — Shin-ansung — Shin-gapyung — Shin-taebaek — Uljin

(a)

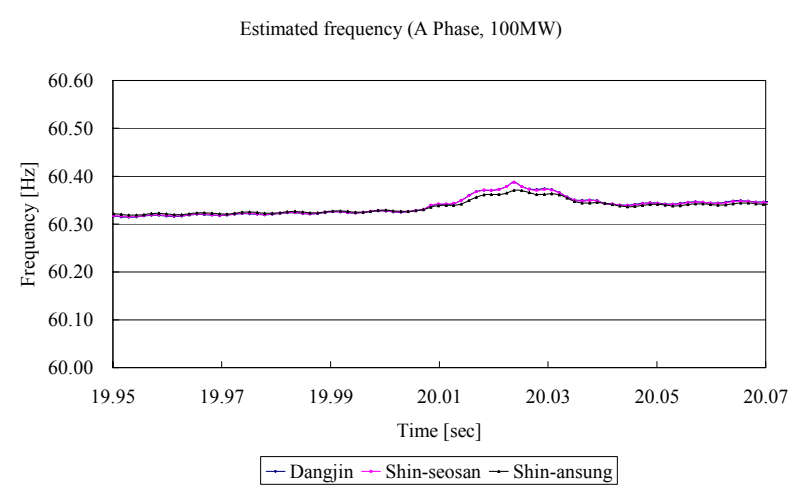

(b)

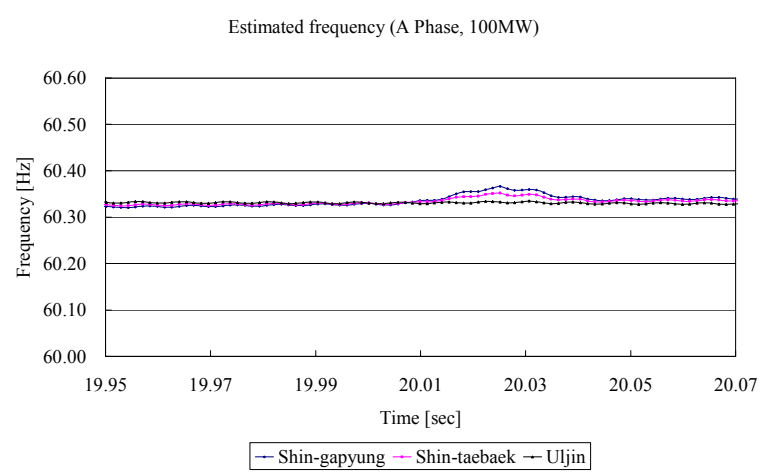

(c)

Fig. 4. Estimated frequency during $100 \mathrm{MW}$ load shedding

frequency by EMTP-RV and the frequency of each local area estimated during $400 \mathrm{MW}$ load shedding at Dangjin. In these cases, inception time of disturbance by load shedding was at approximately $20 \mathrm{sec}$. Using the proposed technique, the frequencies were estimated from the measured voltage data.

Figs. 4(a) and 5(a) show the computed frequency using conventional zero crossing technique in EMTP-RV. Figs. 4(b), 4(c), 5(b), and 5(c) show the computed frequency using the proposed algorithm based on moving window.

In Fig. 4(a), the nominal frequency of the six regions is approximately $60.32 \mathrm{~Hz}$ in normal state. After disturbance

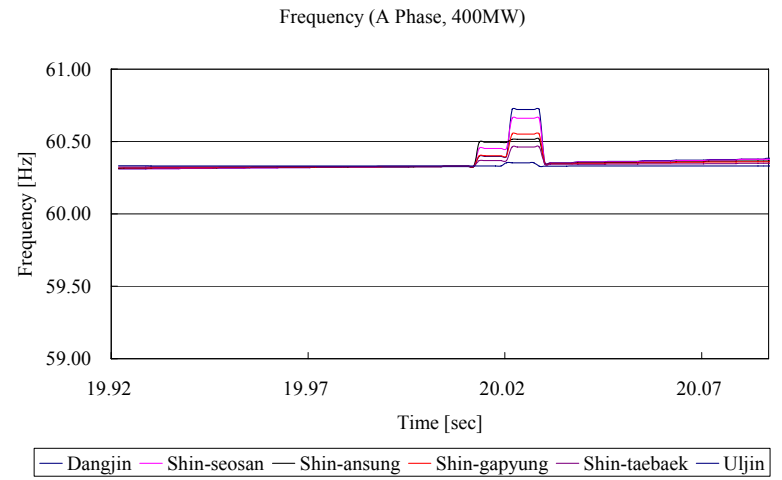

(a)

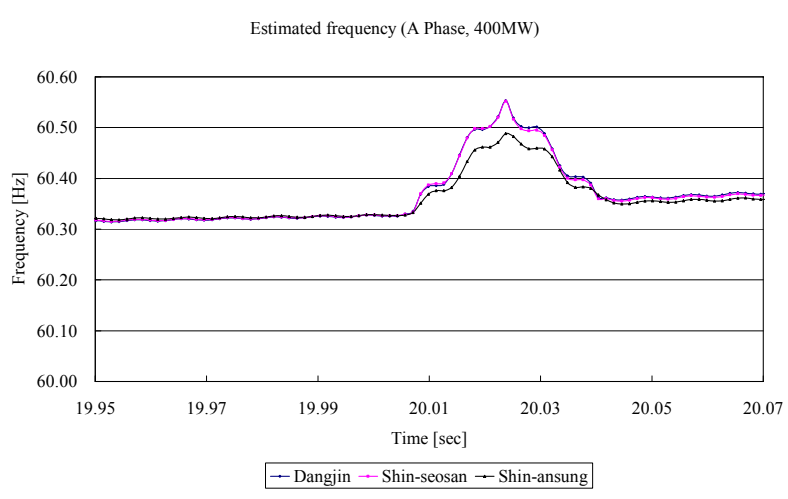

(b)

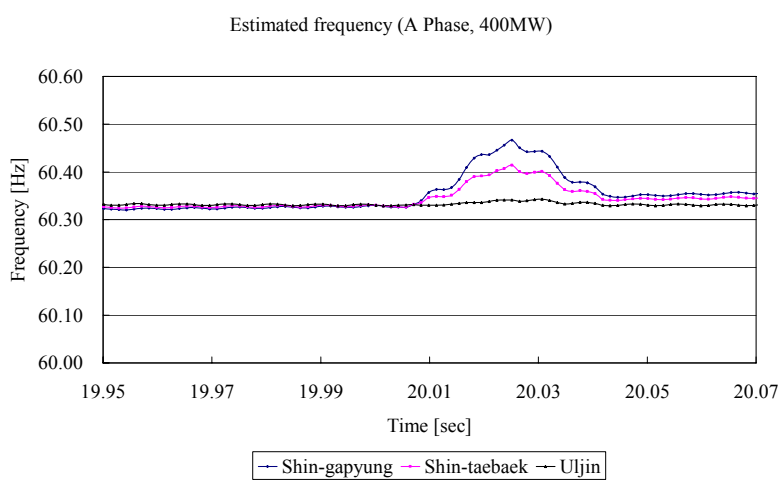

(c)

Fig. 5. Estimated frequency during $400 \mathrm{MW}$ load shedding

occurrence, the frequency of Dangjin T/P is increased to $60.4279 \mathrm{~Hz}$ and subsequently stabilized. In Figs. 4(b) and 4(c), the maximum value of the frequency of the A phase of Dangjin T/P computed using the proposed algorithm is approximately $60.3875 \mathrm{~Hz}$, and the maximum frequency of Shin-seosan S/S is approximately $60.3872 \mathrm{~Hz}$. The maximum frequency of Shin-ansung S/S is about $60.3708 \mathrm{~Hz}$, and the maximum frequency of Shin-gapyung $\mathrm{S} / \mathrm{S}$ is approximately $60.3660 \mathrm{~Hz}$. The maximum frequencies of Shin-taeback S/S and Uljin S/S are approximately 60.3520 $\mathrm{Hz}$, and $60.3344 \mathrm{~Hz}$, respectively.

In Fig. 5(a), the nominal frequency of the six regions is 
approximately $60.32 \mathrm{~Hz}$ in normal state. After disturbance occurrence, the frequency of Dangjin $\mathrm{T} / \mathrm{P}$ increased to 60.7211 Hz prior to stabilization. In Figs. 5(b) and 5(c), the maximum value of frequency of the A phase of Dangjin $\mathrm{T} / \mathrm{P}$ computed using the proposed algorithm is approximately $60.5527 \mathrm{~Hz}$, and the maximum frequency of Shinseosan $\mathrm{S} / \mathrm{S}$ is approximately $60.5520 \mathrm{~Hz}$. The maximum frequency of Shin-ansung S/S is approximately $60.4879 \mathrm{~Hz}$, and the maximum frequency of Shin-gapyung $\mathrm{S} / \mathrm{S}$ is approximately $60.4664 \mathrm{~Hz}$. The maximum frequencies of Shin-taeback S/S and Uljin S/S are approximately 60.4143 $\mathrm{Hz}$, and $60.3432 \mathrm{~Hz}$, respectively.

In Figs. 4 and 5, the magnitude of estimated frequency is increased according to the generating distortion of a voltage soon after the disturbance occurs. The estimated frequency values reveal high accuracy. Generally, the estimated frequency variations decrease according to the distance increments from Dangjin. Likewise, the estimated frequency shows that the higher the load shedding increases, the higher the increase will be in the frequency oscillation.

\subsection{Evaluation using user-defined arbitrary signals}

Seven signals with three conditions are tested to verify the proposed algorithm. The three conditions are the signal change in magnitude, harmonics change, and frequency change. Sampling frequency is $720 \mathrm{~Hz}$.

The first condition is tested against a case where magnitude changes from 0.8 to $1.2 \mathrm{pu}$ in a $0.2 \mathrm{pu}$ step while the frequency and phase angle are unchanged. The result of frequency estimation using the proposed algorithm for the first signal test is shown in Fig. 6. Good frequency estimation with an error less than $\pm 0.33 \mathrm{~Hz}$ can be achieved by the proposed method. The estimated frequency values provide high accuracy after the magnitude change.

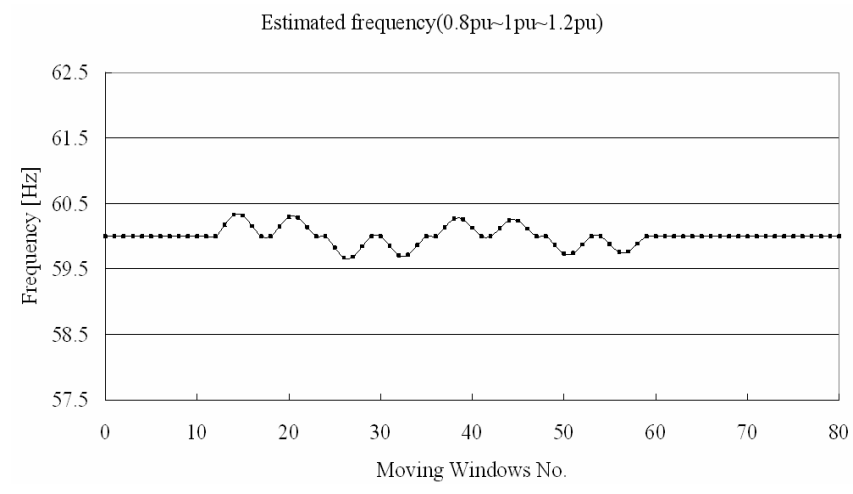

Fig. 6. Estimated frequency during magnitude change

The second condition is tested under the assumption that harmonics change. First, the second harmonic component is assumed to suddenly change from 0 to $0.25 \mathrm{pu}$. Second, the third harmonic component is assumed to suddenly change from 0 to $0.2 \mathrm{pu}$. The estimated results from the proposed algorithm are shown in Fig. 7. Good frequency estimation with an error of less than $\pm 0.1 \mathrm{~Hz}- \pm 0.3 \mathrm{~Hz}$ can be achieved.

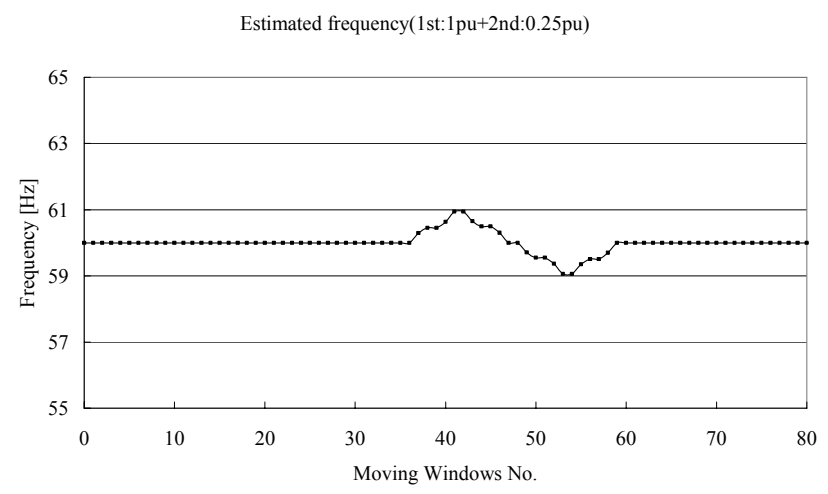

(a)

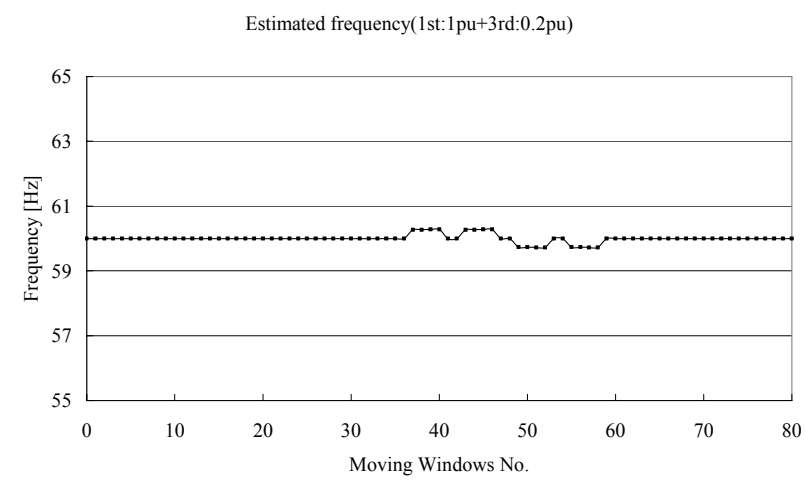

(b)

Fig. 7. Estimated frequency during harmonics change

The third condition is tested against the case where frequency changes from nominal frequency. Fig. 8 shows the frequency estimation results when the frequency is changed from the nominal frequency to various values such as $0.01,0.1,1$, and $6 \mathrm{~Hz}$. In Fig. 8(a), the proposed technique estimates the frequency with a maximum of 0.04 Hz. The estimation, performed after the frequency is changed to $0.01 \mathrm{~Hz}$, resulted in the accurate estimation of, $60.01 \mathrm{~Hz}$ after $39 \mathrm{~ms}$, where $0.01 \mathrm{~Hz}$ is reflected. In Fig. 8(b), the technique estimates the frequency with a maximum of $0.44 \mathrm{~Hz}$. This is conducted after the frequency is changed to $0.1 \mathrm{~Hz}$. The value of, $60.1 \mathrm{~Hz}$ is accurately estimated after two cycles, where $0.1 \mathrm{~Hz}$ is reflected. In Fig. 8(c), the technique estimates the frequency with a maximum of $4.49 \mathrm{~Hz}$ after the frequency is changed to $1 \mathrm{~Hz}$. The value of, $61 \mathrm{~Hz}$ is accurately estimated after two cycles, where $1 \mathrm{~Hz}$ is reflected. In Fig. 8(d), the technique estimates the frequency with a maximum of $14.42 \mathrm{~Hz}$ after the frequency is changed to $10 \%$ of the nominal frequency. Consequently, $66 \mathrm{~Hz}$ is estimated after two cycles, in which $6 \mathrm{~Hz}$ is reflected. The above results confirmed the capability of the proposed 
algorithm to provide good frequency estimation with the error of assumed frequency variation.

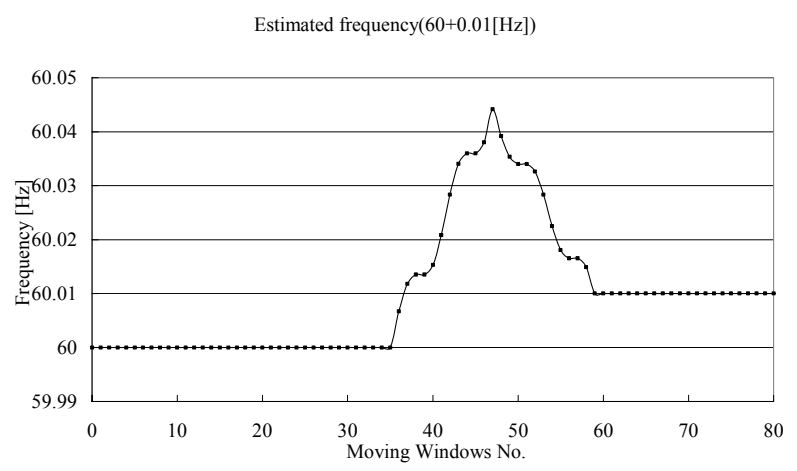

(a)

Estimated frequency $(60+0.1[\mathrm{~Hz}])$

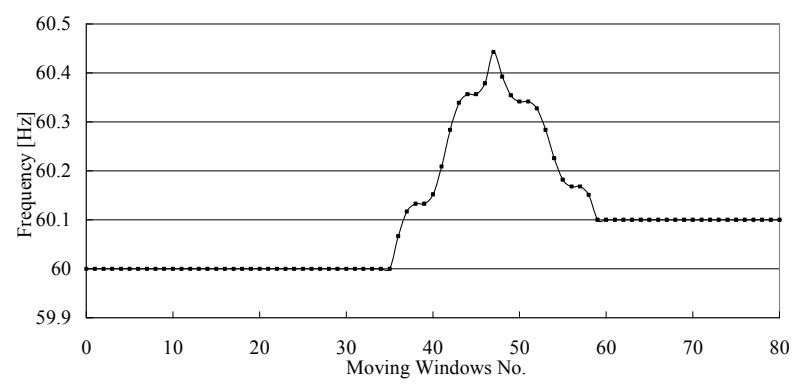

(b)

Estimated frequency $(60+1[\mathrm{~Hz}])$

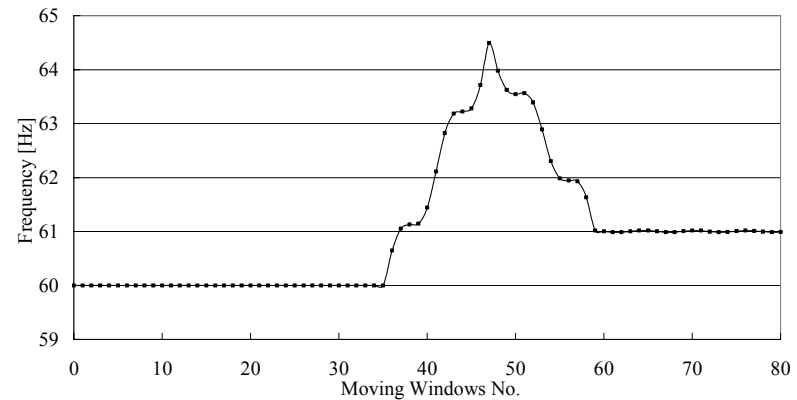

(c)

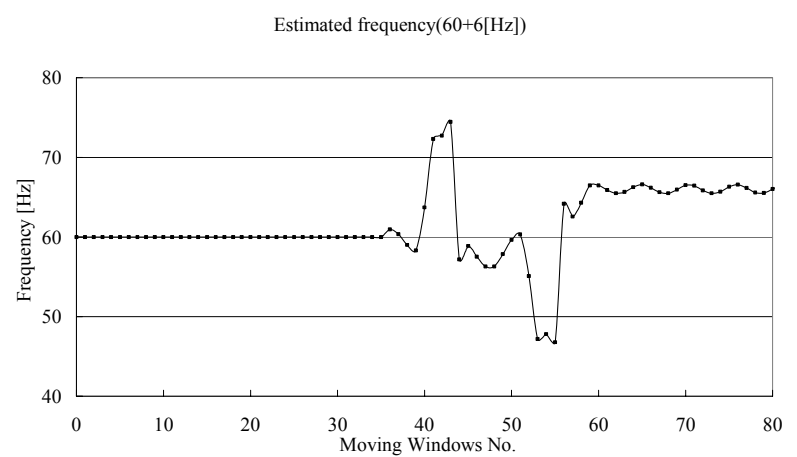

(d)

Fig. 8. Estimated frequency during magnitude change

\section{Conclusion}

An advanced frequency estimation technique using gain compensation was developed aimed to reducing the gain error produced when the frequency deviates from nominal value. For the performance evaluation, we used voltage waveforms obtained from EMTP-RV simulation and userdefined arbitrary signals. The simulation results showed that the proposed technique can provide better accuracy and higher robustness to harmonics and noise under steady state tests and dynamic conditions.

\section{Acknowledgements}

This work was supported by a grant from the Development of Network Based Frequency Monitoring and Fault Prediction System for Wide Area Intelligent Relaying of the Korea Institute of Energy Technology Evaluation and Planning (KETEP) as funded by the Ministry of Knowledge Economy (2008T100100131) of the Korean government.

\section{References}

[1] Chul Won Park, "Development of Network Based Frequency Monitoring and Fault Prediction System for Wide Area Intelligent Relaying”, 2008 Energy $R \& D$ projects, 1 year report, pp. 1-74, 2009.11.

[2] J. Ren, M. Kezunovic, "Use of Resursive Wavelet Transform for Estimating Power System Frequency and Phasors", IEEE PES T\&D Conference, TD2010$000221,2010.4$

[3] C.W. Park, "A Comparative Study of Frequency Estimation Technique using Phasor Angle between Two Phasors and High Speed FIR Filter", KIEE Trans, Vol. 58P, No. 2, pp. 122-129, 2009.6.

[4] A.G. Phadke, "Synchronized Phasor Measurements A Historical Overview", IEEE PES summer meeting, pp. 476-479, 2002.

[5] Zhou Jie, Zhang Changyin, Hw Wei, Jiang Ping, Cheng Chunling, "Precise Measurement of Power System Frequency and Absolute Phase Based on GPS”, pp. 1947-1951, IEEE 2002.

[6] Z. Zhong, A.G. Phadke, Y. Liu et al., "Power System Frequency Monitoring Network (FNET) Implementation", IEEE Trans. on Power Systems, Vol. 20, No. 4, pp. 1914-1921, Nov., 2005.

[7] Lei Wang, Richard W. Conners, Yilu Liu et al., "Frequency Disturbance Recorder Design and Developments", IEEE PES Summer Meeting, 2007.

[8] Sang-Tae Gim, Ji-Yeong Kim, Dong-Cul Yi, WanSeok Song, Su-Hyeong Jang, Young-Don Kim, "Time synchronization using data-area surveillance system implemented in K-WAMS", KIEE Fall Conference, pp. 44-46, 2008.11. 
[9] Young-Hwan Mun, Tae-Hyun Kim "Korean-area power system monitoring system (K-WAMS)", The final report and presentation, pp. 44-46, 2009.8.

[10] Tae-YunGwon, Ll-Kwon Chung, Hyeon-Mo Yi, "Based on satellite communications and GPS data, a synchronous phase-measuring device developed" Proceedings of the PSPES Annual Conference 2008, pp. 101-106, 2008.3.

[11] K.S. Kook1 et al., "Global behaviour of power system frequency in Korean power system for the application of frequency monitoring network", IET Generation Transmission Distribution, Vol. 2, No. 5, pp. 764-774, 2008.

[12] T.S. Sidhu, "Accurate measurement of power system frequency using a digital signal processing technique", IEEE Trans. on I\&M, Vol.48, No.1, pp. 75-81, February, 1999.

[13] C.W. Park, Y.S. Kim, "A Comparative Study of Frequency Estimation Techniques”, IEEE T\&D Asia Conference, PO13-4, Oct., 2009.

[14] P.J. Moore, R.D. Carranza, A.T. Johns, "A new numeric technique for high speed evaluaton of power system frequency", IEE Proc.-Gener. Transm. Distrib., Vol. 141, No. 5, pp. 529-536, Sepember, 1994.

[15] A.G. Phadke, J.S. Thorp, "A new measurement technique for tracking voltage phasor, local system frequency, and rate of change of frequency", IEEE Trans. on PAS., Vol. PAS-102, No. 5, pp. 1025-1034, May, 1983.

[16] S.R. Nam, S.H. Kang, J.K. Park, “An Algorithm for Power Frequency Estimation using the Difference between the Gain of Cosine and Sine Filters", KIEE Trans, Vol. 55A, No. 6, pp. 249-254, Jun., 2006.

[17] C.W. Park, Y.H. Ban, "Advanced Frequency Estimation Algorithm for Frequency Disturbance Recorder", KIEE Spring Conference, PE-O08, pp. 37-39, May, 2010.

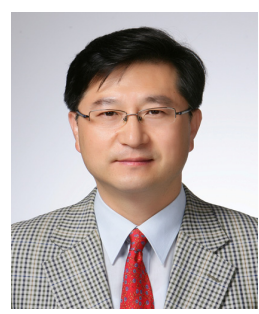

Chul-Won Park was born in Korea in 1961. He received his B.S., M.S. and Ph.D. degrees in Electrical Engineering from Sungkyunkwan University(SKK), Seoul, Korea, in 1988, 1990, and 1996, respectively. From 1989 to 1993 he was an associate researcher at Lucky GoldStar Industrial Systems. From 1993 to 1996, he was a senior researcher at PROCOM system and lecturer at S.K.K. University. At present, he is a professor in the Department of Electrical Engineering at Gangneung-Wonju National University, since 1997. His research interests include power IT, IED, power system modeling and control, and computer application in power system. He is a member of the KIEE. Dr. Park was awarded Paper Prize of KIEE in 2010.

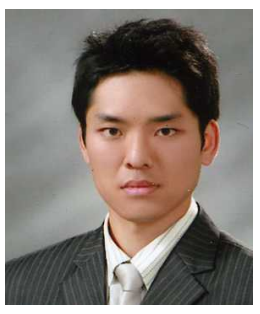

Dong-Kwang Shin was born in Korea in 1981. He received his B.S. degrees from Dongguk University, Seoul, Korea, in 2008. Currently, he is pursuing an M.S. degree at the Sungkyunkwan University, Suwon, Korea. His research interests include power system transients, protection,

and stability.

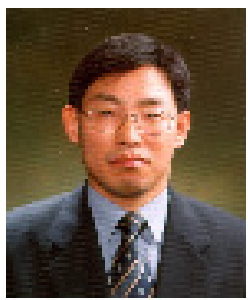

Chul-Hwan Kim (M'90-SM'04) was born in Korea in 1961. He obtained his B.S., M.S., and Ph.D. degrees in Electrical Engineering from Sungkyunkwan University, Suwon, Korea, in 1982, 1984, and 1990, respectively. In 1990, he joined Cheju National University, Cheju, Korea, as a fulltime lecturer. In 1996, 1998, and 1999, he was a visiting academic at the University of Bath, Bath, U.K. Since March 1992, he has been working as professor in the School of Electrical and Computer Engineering, Sungkyunkwan University, where he is also the current Director of the Center for Power IT. His research interests include power system protection, artificial intelligence applications for protection and control, modeling/ protection of underground cables, and EMTP software.

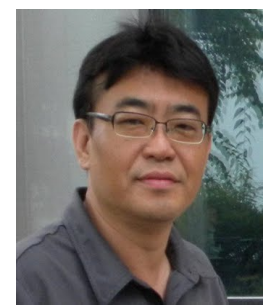

Hak-Man Kim received his Ph.D. degree in Electrical Engineering from Sungkyunkwan University, Korea and his second Ph.D degree in Graduate School of Information Sciences, Tohoku University, Japan. He was a senior researcher of the Korea Electrotechnology Research Institute from October 1996 to February 2008. At present, he is an assistant professor in the Department of Electrical Engineering, University of Incheon, Korea. His research interests include power system modeling and analysis, distributed intelligent power grids and microgrids based on the multiagent system, and IT applications on power grids. Dr. Kim received the Prize of Minister of Science and Technology in 2007, the Outstanding Paper Award from INTELEC 2009, the KIEE Journal Paper Award in 2009, the Best Paper Award from SUCOMS 2010, and the Best Paper Award from UCMA 2011. He is a senior member of KIEE, and is a member of IEEE, IEICE, KIPE, KIIEE, and KIPS. 


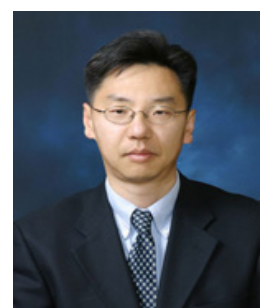

Yoon Sang Kim obtained B.S., M.S., and Ph.D. degrees in Electrical Engineering from Sungkyunkwan University, Seoul, Korea, in 1993, 1995 , and 1999, respectively. He was a member of the Postdoctoral Research Staff of Korea Institute of Science and Technology (KIST), Seoul, Korea. Likewise, he was a Faculty Research Associate in the Department of Electrical Engineering, University of Washington, Seattle. He was a Member of the Senior Research Staff, Samsung Advanced Institute of Technology (SAIT), Suwon, Korea. Since March 2005, he has been an Associate Professor at the School of Computer and Science Engineering, Korea University of Technology Education (KUT), Cheonan, Korea. His current research interests include Virtual simulation, Power-IT technology, and device-based interactive application. Dr. Kim was awarded the Korea Science and Engineering Foundation (KOSEF) Overseas Postdoctoral Fellow in 2000. He is a member of IEEE, IEICE, ICASE, KIPS, and KIEE. 